\title{
Transfer Learning-Based Classification of Breast Cancer using Ultrasound Images
}

\author{
Emek Guldogan ${ }^{1(\underline{I D})}$, Hasan Ucuzal $^{1(\underline{I D})}$, Zeynep Kuçukakcali ${ }^{1(\underline{D})}$ Cemil Colak $^{1(\underline{I D})}$ \\ ${ }^{1}$ Department of Biostatistics and Medical Informatics, Faculty of Medicine, Inonu University, Malatya, \\ Turkey
}

Copyright@Author(s) - Available online at https://dergipark.org.tr/en/pub/mbsjohs

Content of this journal is licensed under a Creative Commons Attribution-NonCommercial 4.0 International

License,

Received: 09 February 2020, Accepted: 08 April 2021, Published online: 30 April 2021

(C) Ordu University Institute of Health Sciences, Turkey, 2021

\begin{abstract}
Objective: One of the most significant cancers impacting the health of women is breast cancer. This study aimed to provide breast cancer classification (benign and malignant) using the transfer learning method on the ultrasound images.

Methods: In the present study, a public imaging dataset was used for the breast cancer classification. Transfer learning technique was implemented for the detection and classification of breast cancer (benign or malignant) based on the ultrasound images. The current research includes data of 150 cases of malignant and 100 normal cases obtained from the Mendeley data. The relevant dataset was partitioned into training (85\% of the images) and validation (15\% of the images) sets. The present study implemented Teachable Machine (teachablemachine.withgoogle.com) for predicting the benign or malignant of breast cancer tumor based on the ultrasound images.

Results: According to the experimental results, accuracy, sensitivity and specificity with $95 \%$ confidence intervals were 0.974 (0.923-1.0), 0.957 (0.781-0.999) and 1 (0.782-1.0), respectively.

Conclusion: The model proposed in this study gave predictions that could be useful to clinicians in classifying breast cancer based on ultrasound images. Thus, this system can be developed in mobile, web, or alternative environments and offered as a computer-aided system for the use of radiologists, pathologists or other healthcare professionals in hospitals.
\end{abstract}

Key words: Breast Cancer, Classification, Ultrasound Images, Transfer Learning.

Suggested Citation: Guldogan E, Ucuzal H, Kucukali Z, Colak C. Transfer Learning-Based Classification of Breast Cancer Using Ultrasound Images..Mid Blac Sea Journal of Health Sci, 2021; 7(1):74-80

\section{Address for correspondence/reprints:}

Emek Guldogan

Telephone number: +905062844934

E-mail: emek.guldogan@inonu.edu.tr 


\section{Introduction}

One of the most significant cancers impacting the health of women is breast cancer. According to global cancer incidence statistics released by the International Agency for Research on Cancer (GLOBOCAN 2018) of the World Health Organization, there are approximately 2.89 million new cases of breast cancer in women worldwide every year, accounting for 24.2 percent of the total cases of female cancer, ranking first (1).

For patients, early detection, diagnosis, and care also generate several benefits. The conventional breast detection approaches to date include breast self-examination, physician palpation, and medical imaging examination. Mammography, ultrasonography, and magnetic resonance imaging are part of the imaging test. Mammography and ultrasonography are among those widely used for the detection of breast tumors. However, mammography sensitivity and precision are still relatively low. In addition, the diagnostic capacity of mammography is inadequate for dense breast and multicenter lesions in patients, in which reduced accuracy contributes to incorrect or missing diagnoses. Ultrasonography has become an essential component of clinical medical exams since it has the advantages of real-time dynamics. Identifying clinically suspicious breast tumors offers vital imaging information and can be used as a screening tool for early, hidden, and noncalcified breast cancer (2). However, the diagnosis of breast cancer ultrasound images is largely dependent on the diagnostic experience of the doctor. Consequently, misdiagnosis and missed diagnosis are easily caused by the lack of qualified ultrasound physicians, immense workload, and exhaustion. In breast cancer diagnosis, computeraided diagnostic systems show great promise (1).

In artificial intelligence, machine learning and computer-aided detection applications, deep convolutional neural networks (CNN) have been of great and widespread use. By monitoring their depth and width, the CNN capabilities can be handled where the precision of assumptions is high for naturebased datasets. But, its utility for classification, segmentation, and identification has been witnessed when it comes to medical imaging. However, certain drawbacks are encountered due to the lack of medical training data from large numbers of images and the absence of deep learning models pre-trained on medical data. In order to help solve those drawbacks, transfer learning is used in two ways. First, it is possible to improve the learning of other layers and change the output size of the end layer by using the pre-trained architecture as a feature extractor that minimizes the dimensionality of the dataset by using it as an input to a narrow classifier, and secondly, by fine-tuning by which layers can be defined to freeze. (3).

This study aimed to provide breast cancer classification (benign and malignant) using the transfer learning method on the ultrasound images.

\section{Methods}

\section{Public dataset}

The current research includes breast cancer ultrasound images of 150 cases of malignant and 100 normal cases obtained from the Mendeley website (4). There is no ground truth for tumor segmentation in this dataset. Thus, manually segmenting tumors in conventional images has been used as a training set for our segmentation model (4).

\section{Transfer learning}

A promising machine learning methodology for solving the above problem is transfer learning, which focuses on transferring knowledge across domains. Initially, the concept of transfer learning may originate from educational psychology. As proposed by psychologist $\mathrm{C}$, according to the generalization theory of transfer. H. Judd, the outcome of the generalization of experience is learning to transfer. A person can realize his transition from one personal situation to another if he generalizes his experiences. According to this theory, communication must be accompanied with connections between learning activities. In practice, since both the violin and the piano are musical instruments, and share some common knowledge, a person who knows how to play the violin will be able to play the piano at an easier speed than others (5). These benefits for transfer learning were emphasized in the "Learning to Learn: Knowledge Consolidation and Transfer in Inductive Systems," post-conference workshop at the NeurIPS conference in December 2012. In that workshop, the implications of transfer learning were discussed in detail. More people have been studying transfer learning techniques since that time. A variety of learning, including the learning of lifetime, inductive transfer, multitask learning, and metalearning have been proposed and applied. Until recently, there was no clear definition of transfer learning (6). There are four types of learning which includes instance based, feature based, parameter based, and relation based (shown in Table 1). Combining different machine learning methods can give better results (6). 
Table 1. Four transfer learning method categories (6)

\begin{tabular}{ll}
\hline \multicolumn{1}{c}{ Technique } & \multicolumn{1}{c}{ Explanation } \\
\hline Instance-based & $\begin{array}{l}\text { Reweighting or selecting highly similar samples (e.g., molecules) from the source } \\
\text { data that match the target data for training support. }\end{array}$ \\
\hline Feature-based & $\begin{array}{l}\text { Identifying the exact features (e.g., molecular fingerprints) between two different } \\
\text { types of molecules. Various techniques can be used to determine target information. }\end{array}$ \\
\hline Parameter-based & $\begin{array}{l}\text { Transfer of parameters (weights) or prior knowledge learned from source data to } \\
\text { target data (e.g., common chemical knowledge). }\end{array}$ \\
\hline Relation-based & Building a map between source and target data for relational knowledge. \\
\hline
\end{tabular}

Inspired by the ability of the human being to transfer knowledge across domains, Transfer Learning aims to leverage knowledge from a domain (known as a source domain) to enhance learning performance or minimize the number of examples of labeling required in a target domain. Not all concepts are always readily transferred or applied successfully. If there is little in common between fields of expertise, there will be no success in the transfer of ideas (5).

\section{Construction of the models}

In the present study, transfer learning approach was used for the classification and detection of breast cancer (benign or malignant) based on the ultrasound images. The relevant dataset was partitioned into training ( $85 \%$ of the images) and validation (15\% of the images) sets. In order to better understand the model's results, the model was trained and tested using the training data and the test data of the datasets individually. The present study implemented Teachable Machine (teachablemachine.withgoogle.com) for predicting the benign or malignant breast cancer tumor based on the ultrasound images.

Teachable Machine is a web application that allows any user to train their own machine learning models without technical expertise. Since it uses ANN, a simple model is easy to classify the images. Users can add new information to a model and the model can then be trained on top of a previously trained model. The feature extractor was trained to recognize 1000 classes for the portion of the image of Teachable Machine (like a dog, phone, bed, trombone, etc.). This model can be used for identifying new classes created by the user. The data is hidden from the end-users who benefit from simple models with minimal data and training time (7). Teachable Machine offers a training panel for those who prefer more control over model training process. The learning mode advanced could be extended to include an epoch, batch size, and learning rate. For training image classification models, the default parameters to all-new image classification projects although most users will never need to tweak them for satisfactory results (7). In the current study, all possible combinations of three hyper-parameters: epoch (1 to 100), batch size (16, 32, 64 and 128), and learning rate $(0.01$ and 0.00$)$ were tested for best The graphical representation for the various performance metrics of the proposed model is plotted in Figure 1.

performance metrics. Epoch, batch size, and learning rate, were selected to be 50, 16, and 0.001, respectively.

\section{Evaluation metrics}

The current research assessed the proposed model regarding many performance metrics calculated from the confusion matrix. Our DTROC software calculates accuracy, sensitivity, specificity, positive/negative predictive values, F1 score, Youden's index, Mathew's correlation coefficient (MCC), and so on (8). We reported all appropriate classification metrics using DTROC software (8) in this study.

\section{Results}

From the construction of the proposed approach, the classification matrix of the test samples for the transfer learning model used to classify breast cancer is shown in Table 2 .

Table 2. Classification matrix of the test samples for transfer learning model

\begin{tabular}{|c|c|c|c|}
\hline $\begin{array}{c}\text { True } \\
\text { Prediction } \\
\end{array}$ & Positive & Negative & Total \\
\hline Positive & 22 & 0 & 23 \\
\hline Negative & 1 & 15 & 15 \\
\hline Total & 23 & 15 & 38 \\
\hline
\end{tabular}


Accuracy, F1-Score, MCC, prevalence, true prevalence, sensitivity, specificity, diagnostic accuracy, Youden's index, positive predictive value, and negative predictive value for transfer learning model are summarized together with $95 \%$ confidence intervals for test samples in Table 3. According to the experimental results, accuracy, sensitivity and specificity with $95 \%$ confidence intervals were 0.974
(0.923-1.0), 0.957 (0.781-0.999) and 1 (0.782-1.0), respectively.

The graphical representation for the various performance metrics of the proposed model is plotted in Figure 1

Table 3. Performance Metrics of the proposed model on test samples

Metrics

Estimated Value 95\% Confidence interval
for lower limit

\begin{tabular}{lccc}
\hline Accuracy & 0.974 & 0.923 & 1.0 \\
F1-Score & 0.978 & 0.931 & 1.0 \\
MCC & 0.947 & 0.876 & 1.0 \\
Prevalence & 0.579 & 0.408 & 0.737 \\
True Prevalence & 0.605 & 0.434 & 0.76 \\
Sensitivity & 0.957 & 0.781 & 0.999 \\
Specificity & 1.0 & 0.782 & 1.0 \\
Diagnostic Accuracy & 0.974 & 0.862 & 0.999 \\
Youden's index & 0.957 & 0.562 & 0.999 \\
Positive Predictive Value & 1.0 & 0.846 & 1.0 \\
Negative Predictive Value & 0.938 & 0.698 & 0.998 \\
\hline
\end{tabular}

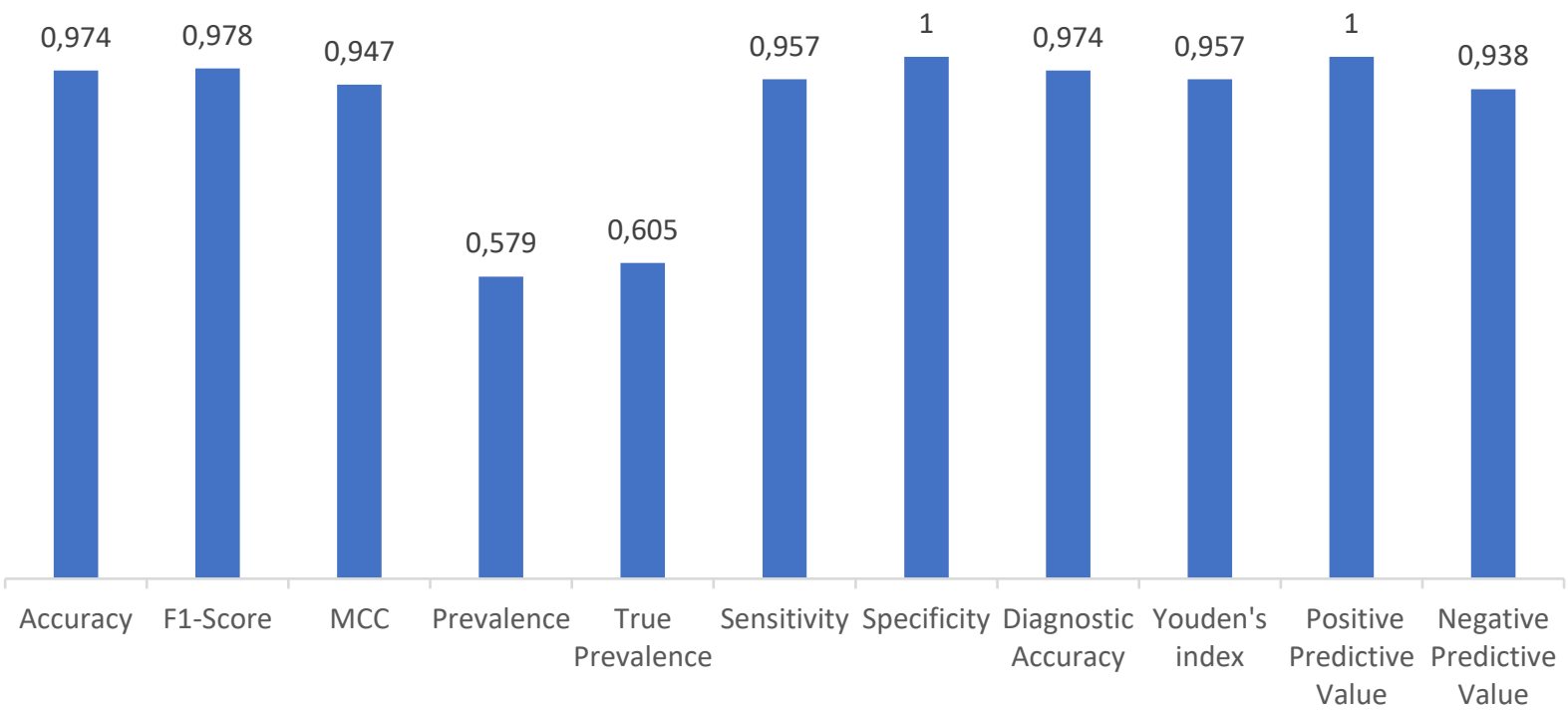

Figure 1. Graphical representation for the various performance metrics of the proposed model 


\section{Discussion}

Cancer is a disease in which cells multiply, crowding out other cells. One of the most common types of cancer disease is breast cancer. Breast cancer is the most common type of cancer in women. Due to late diagnosis and ignorance of the disease, many women die from breast cancer. Breast cancer detection systems based on female imagery have gained popularity in recent years. In recent years, Deep Learning has attracted a lot of researchers, and a great variety of computer vision applications have appeared in a wide variety of environments. The use of computer programs to help diagnose breast cancer has significantly eased the burden on doctors (9). To construct a computer-aided system for the diagnosis of breast cancer, this study was carried out to construct a model for the classification of breast cancer from ultrasound images using the transfer learning approach.

Transfer learning is defined as transferring to another domain for classification, and feature extraction purposes of knowledge learned earlier in one domain. Transfer learning is performed in the deep learning perspective by using a deep convolutional neural network (CNN) previously trained on a large dataset. The $\mathrm{CNN}$ pre-trained model is further trained (fine-tuned) on a new dataset with a smaller number of training images comparable to the previously trained datasets. Transfer learning has recently become popular among deep learning applications because it is faster and easier than training a pre-trained $\mathrm{CNN}$ model. We recognize basic special characteristics, like edges, corners, curves, and blobs. Only the last three layers are a major requirement in the practical applications and the remaining stages are applied for classification (10). There has been a large amount of studies in recent years on the widespread use of transfer learning techniques to predict and classify different cancer diseases in medicine and other health fields (11-14). A recent paper suggests a machine learningbased approach to automate breast cancer classification from histopathological images using the deep neural network ResNet-18. The experimental findings on the publicly available BreaKHis dataset show that the approach is promising and efficient, outperforming recent stateof-the-art magnification dependent and magnification independent counterparts by a fair margin (15). Another up-to-date work focuses primarily on the process of transfer learning for detecting breast cancer on datasets of 2D and 3D mammogram images through transfer learning models. Experimental results demonstrated that a $94.3 \%$ accuracy is provided by the proposed hybrid transfer learning model (a fusion of Modified VGG and ImageNet), and a precision of 89.8 percent is only provided by the proposed MVGG architecture (16). In a recently published paper, to overcome the existing limitations regarding medical image detection, the model uses real-time data processing augmentation and transfer learning. The results showed that the Xception model trained using transfer learning provided the best results (with 90.86 percent accuracy on the classification task), exceeding the results previously obtained on the BreakHis dataset by the state-of-theart system. (17). The proposed model in the present study classified benign and malignant breast cancer with very high predictive values on the ultrasound images. A new algorithm developed by a study makes use of deep learning algorithms to detect and classify pathological characteristics of breast cancer. Transferred learning attempts to transfer knowledge from one problem to another problem. Feature Extraction uses pre-trained feature extraction models, namely VGGnet and ResNet, which include features for fully connected layers which classified images into benign or malignant cells. This proposed system has shown to be quite effective, and accuracy rate of the proposed system is very high (18). In another study, a convolutional neural network approach to extract the best features from the Breast Imaging dataset to diagnose breast cancer. The experiments in this study were measured by a magnification factor of 4. (40X, 100X, 200X and 400X). Each image is a picture. The network model was trained and validated on $80 \%$ of the work images and $20 \%$ of the test images. The system has achieved accuracy, sensitivity, specificity, and AUC values of 95, 97, 90, and 99.36 respectively. Although the number of images in the target data set (BreaKHis) is not large, AlexNet achieved superior results with the four magnification factors (19). The accuracy rate from this study was higher than other studies on the same topic previously described. This result reveals that the proposed architecture in this study is very useful for predicting benign or malignant breast cancer and can be used by clinicians as a computer-aided diagnosis system. However, in order to test the external validity of the predictions of the proposed classification model, more ultrasound images of breast cancer are necessary for achieving robust results.

There was a class imbalance problem for the breast cancer ultrasound images used in this study. Yet, performance metrics of the proposed transfer learning model for classifying breast cancer may not have been affected by the class imbalance problem, and quite high estimation results (e.g., F1- 
Score $=0.978 ; \mathrm{MCC}=0.947$ ) were obtained from this study. In order to solve the class imbalance issue not implemented in this study, various techniques have been proposed for image processing and classification processes (20-22). If the desired level of classification results is not obtained and there is a class imbalance, it may be very useful to apply hyperparameter optimization and similar approaches to solve the class imbalance in such cases.

\section{Conclusions}

In summary, the model proposed in this study gave predictions that could be useful to clinicians in classifying breast cancer based on ultrasound images. Thus, this system can be developed in mobile, web, or alternative environments and offered as a computer-aided system for the use of radiologists, pathologists, or other healthcare professionals in hospitals

Ethics Committee Approval: Committee Approval Certificate is not required for this study.

Peer-review: Externally peer-reviewed.

Author Contributions:

Concept: E.G, H.U, Design: E.G, C.C; Literature search: E.G, Z.K, Data Collection and Processing: E.G. H.U, Z.K, Analysis or Interpretation: E.G, C.C, Writing: E.G. H.U, Z.K, C.C

Conflict of Interest: No conflict of interest was declared by the authors.

Financial Disclosure: The authors declared that this study hasn't received no financial support.

\section{References}

1. Zhang X, Lin X, Zhang Z, Dong L, Sun X, Sun D, et al. Artificial intelligence medical ultrasound equipment: application of breast lesions detection. Ultrasonic Imaging. 2020;42(4-5):191-202.

2. Zhuang Z, Kang Y, Joseph Raj AN, Yuan Y, Ding W, Qiu S. Breast ultrasound lesion classification based on image decomposition and transfer learning. Medical Physics. 2020.

3. Hijab A, Rushdi MA, Gomaa MM, Eldeib A, editors. Breast cancer classification in ultrasound images using transfer learning. 2019 Fifth International Conference on Advances in Biomedical Engineering (ICABME); 2019: IEEE.

4. Rodrigues PSJMD. Breast ultrasound image. 2017;1.

5. Zhuang F, Qi Z, Duan K, Xi D, Zhu Y, Zhu H, et al. A comprehensive survey on transfer learning. 2020;109(1):43-76.
6. Cai C, Wang S, Xu Y, Zhang W, Tang K, Ouyang $\mathrm{Q}$, et al. Transfer Learning for Drug Discovery. Journal of Medicinal Chemistry. 2020;63(16):8683-94.

7. Carney M, Webster B, Alvarado I, Phillips K, Howell N, Griffith J, et al., editors. Teachable machine: Approachable Web-based tool for exploring machine learning classification. Extended Abstracts of the $2020 \mathrm{CHI}$ Conference on Human Factors in Computing Systems; 2020.

8. YAŞAR Ş, ARSLAN A, Colak C, Yoloğlu SJMBSJoHS. A Developed Interactive Web Application for Statistical Analysis: Statistical Analysis Software.6(2):227-39.

9. Deniz E, Şengür A, Kadiroğlu Z, Guo Y, Bajaj V, Budak ÜJHis, et al. Transfer learning based histopathologic image classification for breast cancer detection. 2018;6(1):1-7.

10. Krizhevsky A, Sutskever I, Hinton GEJCotA. ImageNet classification with deep convolutional neural networks. 2017;60(6):84-90.

11.Abbasi AA, Hussain L, Awan IA, Abbasi I, Majid A, Nadeem MSA, et al. Detecting prostate cancer using deep learning convolution neural network with transfer learning approach. 2020;14(4):52333.

12.Celik Y, Talo M, Yildirim O, Karabatak M, Acharya URJPRL. Automated invasive ductal carcinoma detection based using deep transfer learning with whole-slide images. 2020;133:2329.

13. Chaves E, Gonçalves CB, Albertini MK, Lee S, Jeon G, Fernandes HCJAO. Evaluation of transfer learning of pre-trained $\mathrm{CNNs}$ applied to breast cancer detection on infrared images. 2020;59(17):E23-E8.

14. Khamparia A, Singh PK, Rani P, Samanta D, Khanna A, Bhushan BJToETT. An internet of health things-driven deep learning framework for detection and classification of skin cancer using transfer learning. 2020:e3963.

15.Boumaraf S, Liu X, Zheng Z, Ma X, Ferkous CJBSP, Control. A new transfer learning based approach to magnification dependent and independent classification of breast cancer in histopathological images. 2021;63:102192.

16. Khamparia A, Bharati S, Podder P, Gupta D, Khanna A, Phung TK, et al. Diagnosis of breast cancer based on modern mammography using hybrid transfer learning. 2021:1-19. 
17.Rai R, Sisodia DS. Real-time data augmentation based transfer learning model for breast cancer diagnosis using histopathological images. Advances in Biomedical Engineering and Technology: Springer; 2021. p. 473-88.

18. Khan S, Islam N, Jan Z, Din IU, Rodrigues JJCJPRL. A novel deep learning based framework for the detection and classification of breast cancer using transfer learning. 2019;125:1-6.

19.Senan EM, Alsaade FW, Al-mashhadani MIA, Theyazn H, Al-Adhaileh MHJJoAS, Engineering. Classification of Histopathological Images for Early Detection of Breast Cancer Using Deep Learning. 2021;24(3):323-9.

20.Bria A, Marrocco C, Tortorella FJCib, medicine. Addressing class imbalance in deep learning for small lesion detection on medical images. 2020;120:103735.

21.Zhang C, Tavanapong W, Kijkul G, Wong J, De Groen PC, Oh J, editors. Similarity-based active learning for image classification under class imbalance. 2018 IEEE International Conference on Data Mining (ICDM); 2018: IEEE.

22. Gao L, Zhang L, Liu C, Wu SJAIiM. Handling imbalanced medical image data: A deep-learningbased one-class classification approach. 2020;108:101935. 